\title{
Synthesis and Characterization of Acid Doped Polyaniline for Super Capacitor Application
}

\author{
Santosh Bhattarai ${ }^{1}$, Nim Bhadur Devkota ${ }^{1}$, Shova Neupane ${ }^{1}$, Dipak Kumar Gupta ${ }^{2 *}$, Amar Prasad \\ Yadav $^{1}$ \\ ${ }^{1}$ Central Department of Chemistry, Tribhuvan University, Kirtipur, Kathmandu, Nepal ${ }^{1}$ \\ ${ }^{2}$ Department of Chemistry, Tri-chandra Multiple Campus, Kathmandu, Nepal ${ }^{2}$ \\ E-mail:deepakguptas2012@yahoo.com
}

\begin{abstract}
Polyaniline (PANi) was synthesized from double distilled aniline solution dissolved in $\mathrm{H}_{2} \mathrm{SO}_{4}$ solution by chemical oxidative polymerization method using ammonium persulfate (APS) as an oxidant at room temperature. The four PANi samples were prepared by different acids like $\mathrm{H}_{2} \mathrm{SO}_{4}, \mathrm{HClO}_{4}, \mathrm{H}_{3} \mathrm{PO}_{4}$ and $\mathrm{CH}_{3} \mathrm{COOH}$ at room temperature. Composites of various sample of PANi were made with multiwall carbon nanotube and paraffin for the preparation of electrode to study electrochemical properties such as $\mathrm{CV}$ and charge discharge cycle. The $\mathrm{CV}$ curve shows that sample $\mathrm{PANi}_{2} \mathrm{H}_{2} \mathrm{SO}_{4}$ has highest specific capacitance in comparison to other samples and also the charge discharge cycle shows highest specific capacitance for the sample PANi- $\mathrm{H}_{2} \mathrm{SO}_{4}$ at potential range -0.8 to 1.2 with scan rate 0 f $50 \mathrm{mVs}^{-1}$. PANi samples were characterized by FTIR, XRD and UV-Visible spectroscopy also supports the formation of PANi. Based on the properties shown PANi- $\mathrm{H}_{2} \mathrm{SO}_{4}$ would be the best material for pseudocapacitor application.
\end{abstract}

Keywords: Specific Capacitance (SC), Super Capacitor, MWCNTs, Cyclic voltammetry.

\section{Introduction}

Supercapacitors are important energy storage devices having higher energy density and higher power density compared to batteries and conventional capacitors. Depending on the charge storage mechanism, it can be classified in two categories. The electrical double layer capacitor utilizes mainly the separation of the electronic and ionic charges at the interface between electrode materials and the electrolyte solution. Another type of supercapacitor, referred to as a pseudocapacitor, provides its capacitance from faradic redox reactions occurring within the active materials of electrode ${ }^{1}$. Supercapacitors have attracted increasing interests due to their high power density, longer cycle life, and low maintenance compared to conventional batteries. Electronically conducting polymers like as polypyrrole, polyaniline (PANi), polythiophene and poly [3, 4 ethylenedioxythiophene] can store and release charges through redox processes associated with the $\pi$-conjugated polymer chains. When oxidation occurs, ions from the electrolyte were transferred to the polymer backbone ${ }^{2}$.

One of the important polymers, polyaniline (PANi) has attracted much attention because of their low cost, environmental stability, controllable electrical conductivity, and easy process ability. Combined

\footnotetext{
* Corresponding author
} 
PANi with various robust carbon materials such as graphite oxide, graphene, carbon nanotubes (CNTs), carbon fiber and mesoporous carbon, seems a promising way for the enhanced electrochemical properties. In addition, the stability, electrical conductivity, and redox behavior of PANi could be greatly improved when incorporated with conductive carbon materials ${ }^{3}$.

Our aim is to develop the super capacitor performance and increase the cyclic stability of supercapacitor electrode material. For this purpose we have used multiwall carbon nano tube which has high surface area, low resistance, high electron donor and acceptor properties and high stability indicates that it can be used to improve the supercapacitive performances and stability of the supercapacitor electrode materials ${ }^{4}$.In this study, we synthesized and characterized the PANi composite with acidic dopant by using multiwall carbon nanotube and their electrochemical properties have been compared with different samples ${ }^{5}$.

In this work we have prepared $\mathrm{PANi}$ by the chemical oxidation method at room temperature and studied the effect of different dopants like $\mathrm{H}_{2} \mathrm{SO}_{4}, \mathrm{HClO}_{4}, \mathrm{H}_{3} \mathrm{PO}_{4}$ and $\mathrm{CH}_{3} \mathrm{COOH}$ on the structure of the polymer. The prepared PANi was characterized by FTIR, UV-visible and XRD techniques.

\section{Experimental Methods}

\section{Preparation of Polyaniline (PANi) by Chemical Oxidation Method.}

$0.25 \mathrm{M}$ aniline sulphuric acid solution and $0.31 \mathrm{M}$ ammonium persulphate (APS) were prepared at room temperature was mixed by adding APS from burette drop wise (about 1 drop per second) into aniline solution. Next day the resulting dark green PANi precipitate was collected in a whatman filter paper then washed with distilled water until the filtrate become colorless. It was followed by $0.32 \mathrm{M}$ $\mathrm{H}_{2} \mathrm{SO}_{4}$ then ethanol. Eemeraldine salt precipitate was dried then sample was kept in air tight borosil glass bottle. The sample was dedoped by using the excess of ammonia solution. $0.32 \mathrm{M}$ solution of the respective acids $\left(\mathrm{H}_{2} \mathrm{SO}_{4}, \mathrm{HClO}_{4}, \mathrm{H}_{3} \mathrm{PO}_{4}\right.$ and $\left.\mathrm{CH}_{3} \mathrm{COOH}\right)$ were added in prepared PANi then magnetically stirred, filtered, washed then dried. PANi perared by chemicaloxidation method doped with different acids were separately mixed with MWCNT. PANi/MWCNT ratio was 4:1 in DMF solvent.

All the prepared samples were characterized by UV-VIS, FTIR and XRD methods. UV visible spectra of all samples were recorded by using GENESIS-10 UV-visible pectrophotometer of Thermo Fischer at room temperature in the range of wavelength 300 to 900 nanometer (nm), FTIR spectra of all samples were recorded by using IRTracer-100 of SHIMADZU spectrophotometer as $\mathrm{KBr}$ pellet at room temperature in the range of $4000-400 \mathrm{~cm}^{-1}$ and XRD pattern of all samples were recorded by using diffractometer $\mathrm{D}_{2}$-Phaser of Bruker $\mathrm{GmBH}\left(\mathrm{CuK}_{\alpha}\right.$ radiation, Wavelength $\left.1.5406 \mathrm{~nm}\right)$ at room temperature in the range of $2 \theta$ throughout $10-80^{\circ}$ at Nepal Academy of Science and Technology (NAST), Lalitpur.

\section{Results and Discussion}

\section{Chemical Polymerization of Aniline}

Aniline was chemically polymerized by using oxidizing agent ammonium persulphate $\left[\left(\mathrm{NH}_{4}\right)_{2} \mathrm{~S}_{2} \mathrm{O}_{8}\right]$ in acidic medium $\left(\mathrm{H}_{2} \mathrm{SO}_{4}\right)$. Polymerization of aniline was done at room temperatures. PANi (ES) was synthesized and de-doped with the excess of ammonia solution and again doped separately with various acids like $\mathrm{H}_{2} \mathrm{SO}_{4}, \mathrm{CH}_{3} \mathrm{COOH}, \mathrm{H}_{3} \mathrm{PO}_{4}$ and $\mathrm{HClO}_{4}$. The composites of PANi were formed by mixing MWCNT. 


\section{Capacitive study}

CV of various samples was plotted by using HOKUTO DENKO HA-151 Potentiostat/Galvanostat controlled by Polarization software interfaced with IBM computer.The capacitive characteristic of the PANi-MWCNT composites were investigated by means of the cyclic voltammatry. Capacitance was calculated from the obtained cyclic voltammogram by dividing average current with scan rate while specific capacitance was calculated by dividing capacitance by weight of the active material.

Table 1: Specific Capacitance of PANi from CV

\begin{tabular}{|c|c|}
\hline Samples of PANi & Specific Capacitance $\left(\mathrm{Fg}^{-1}\right)$ \\
\hline PANi- $\mathrm{H}_{2} \mathrm{SO}_{4}$ & 362.12 \\
\hline $\mathrm{PANi}^{-\mathrm{HClO}_{4}}$ & 301.79 \\
\hline $\mathrm{PANi}^{2} \mathrm{H}_{3} \mathrm{PO}_{4}$ & 241.43 \\
\hline PANi- $\mathrm{CH}_{3} \mathrm{COOH}$ & 281.67 \\
\hline
\end{tabular}

(a)

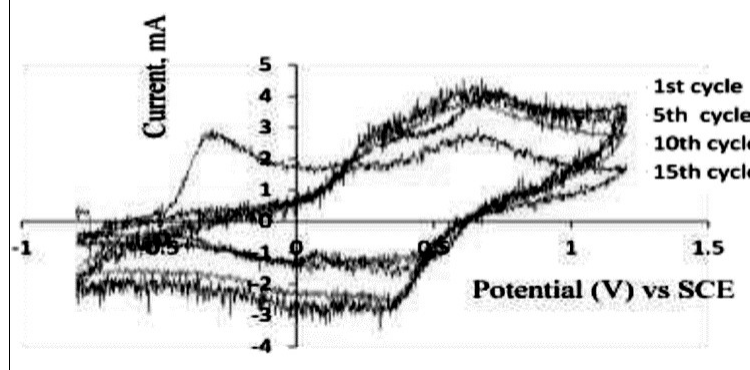

(c)

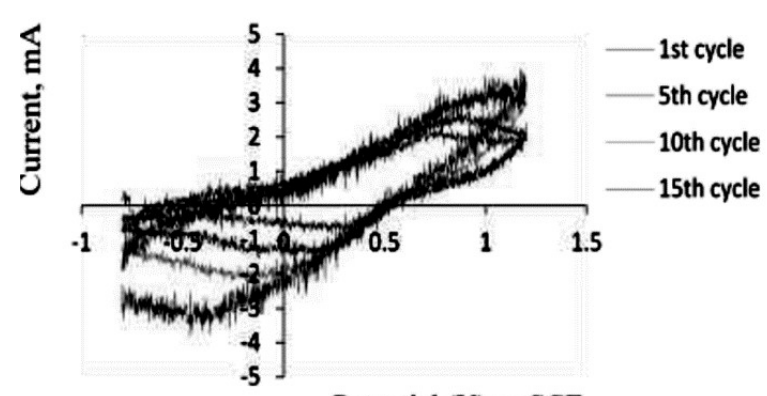

Potential (V) vs SCE (b)

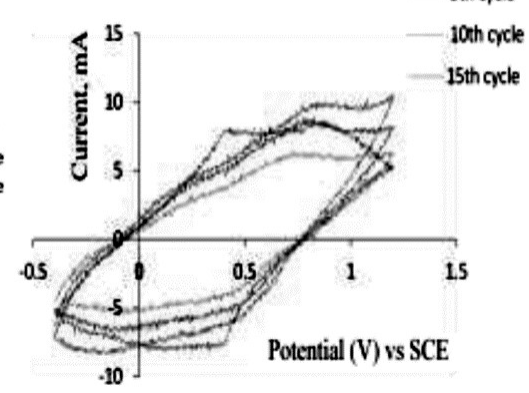

(d)

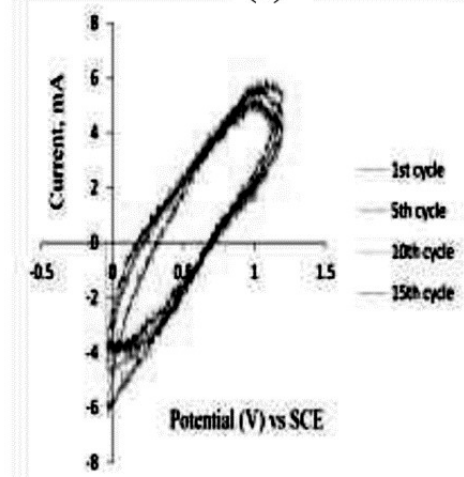

Figure 1: Cyclic Voltammetry of samples(a) $\mathrm{PANi}_{-} \mathrm{H}_{2} \mathrm{SO}_{4}$ (b) $\mathrm{PANi}-\mathrm{HClO}_{4}$ (c) $\mathrm{PANi}-\mathrm{H}_{3} \mathrm{PO}_{4}$ (d) $\mathrm{PANi}$ $\mathrm{CH}_{3} \mathrm{COOH}$

The specific capacitance for sample PANi- $\mathrm{H}_{2} \mathrm{SO}_{4}$ was found to be maximum $\left(362.12 \mathrm{~F} \mathrm{~g}^{-1}\right)$ while minimum $\left(241.43 \mathrm{Fg}^{-1}\right)$ for $\mathrm{PANi}^{-} \mathrm{H}_{3} \mathrm{PO}_{4}$ 


\section{Galvanostatic Charge- Discharge Curve}

The galvanostatic charge discharge cycle of sample composites were at a current density of $10 \mathrm{~mA}$ $\mathrm{cm}^{-2}$ in three electrode system between the potential ranges of -0.8 to $1.2 \mathrm{~V}$ was performed by using HOKUTO DENKO HA-151 Potentiostat/Galvanostat controlled by 2 ch-Monitor software interfaced with IBM computer.
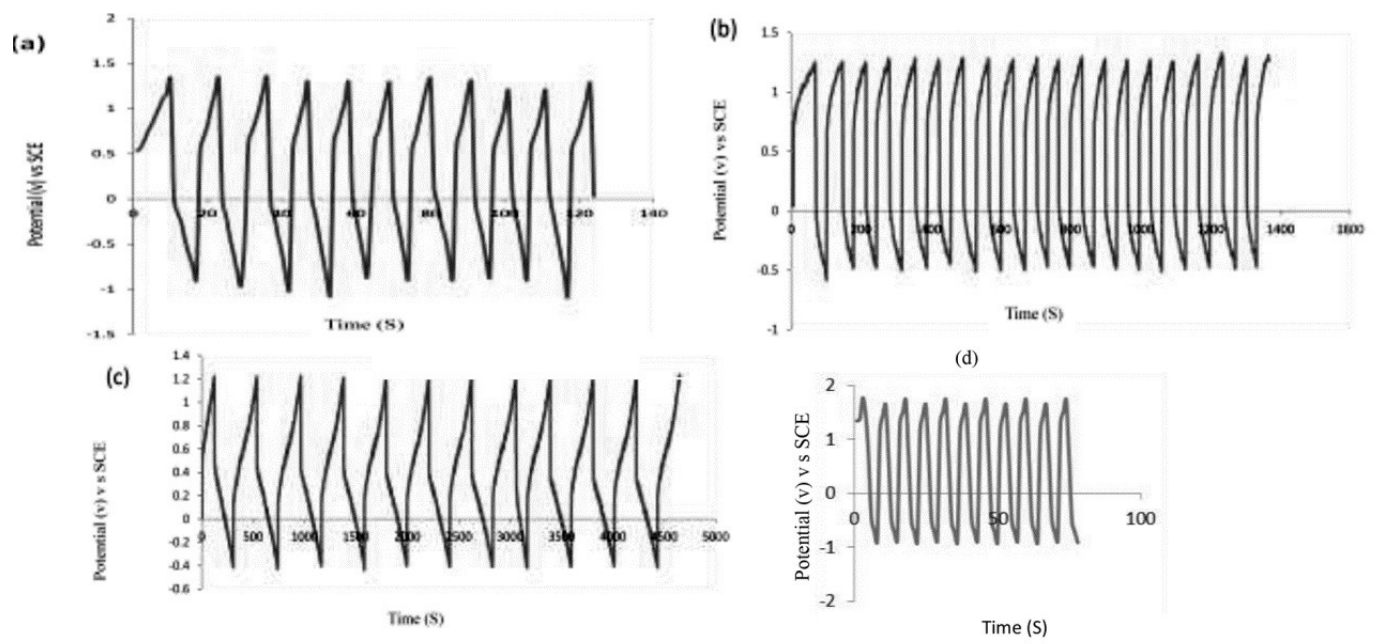

Figure 2: Charge discharge curve of the composite materials (a) $\mathrm{H}_{2} \mathrm{SO}_{4}$ (b) $\mathrm{HClO}_{4}$ (c) $\mathrm{H}_{3} \mathrm{PO}_{4}$ (d) $\mathrm{CH}_{3} \mathrm{COOH}$

Table2: Specific Capacitance of the PANi samples obtained from charge discharge curve.

\begin{tabular}{|c|c|}
\hline Samples of PANi & Specific Capacitance $\left(\mathrm{Fg}^{-1}\right)$ \\
\hline PANi- $\mathrm{H}_{2} \mathrm{SO}_{4}$ & 382.29 \\
\hline $\mathrm{PANi}_{-} \mathrm{HClO}_{4}$ & 202.12 \\
\hline PANi- $\mathrm{H}_{3} \mathrm{PO}_{4}$ & 106.38 \\
\hline $\mathrm{PANi}-\mathrm{CH}_{3} \mathrm{COOH}$ & 191.48 \\
\hline
\end{tabular}

The specific capacitance values of all the composite determined using the galvanostatic charge discharge method followed the trend determined by the $\mathrm{CV}$ measurements. SC was found to maximum for the PANi sample doped with $\mathrm{H}_{2} \mathrm{SO}_{4}$ at room temperature. It was observed that $\mathrm{SC}$ of $\mathrm{PANi}-\mathrm{H}_{3} \mathrm{PO}_{4}$ is less in comparison to other PANi samples.

\section{Characterization of PANi}

The UV-visible spectra of the PANi sample were recorded by using DMF solution. PANi shows two absorption bands curves: one location is about $350 \mathrm{~nm}$ and the other is about $590 \mathrm{~nm}$.FTIR shows the band at about $798 \mathrm{~cm}^{-1}$ for N-H wag of secondary amines (1 hydrogen and 2 aromatic groups). The band at about $1296 \mathrm{~cm}^{-1}$ is assigned to the $\mathrm{C}-\mathrm{N}$ stretching mode of the aromatic amines benzenoid units. 
Characteristic bands at about $1559 \mathrm{~cm}^{-1}$ and $1466 \mathrm{~cm}^{-1}$ corresponding to the $\mathrm{C}=\mathrm{C}$ and $\mathrm{C}-\mathrm{C}$ stretching vibration of the quinoid and benzenoid structure respectively. It should be noted that the quinoid vibration intensity is appeared to be less than that of the benzenoid vibration in pure PANi ES. The peak $\sim 671 \mathrm{~cm}^{-1}$ is attributed to the out-of-plane bending of $\mathrm{C}-\mathrm{H}$. The band at $\sim 1125-1148 \mathrm{~cm}^{-1}$ is assigned to plane bending vibration of $\mathrm{C}-\mathrm{H}$, which was formed during protonation of the PANi chain. The peak at $3460 \mathrm{~cm}^{-}$ ${ }^{1}$ and $2330 \mathrm{~cm}^{-1}$ can be attributed to the free (non hydrogen bonded) $\mathrm{N}-\mathrm{H}$ stretching vibration and hydrogen bonded $\mathrm{N}-\mathrm{H}$ bond between amine and imine sites. XRD patterns of PANi samples shows broad diffraction peaks occur between at $16^{\circ}, 26^{\circ}$ and $42^{\circ}$ due to the parallel and perpendicular periodicity of the polymer (PANi) chain.

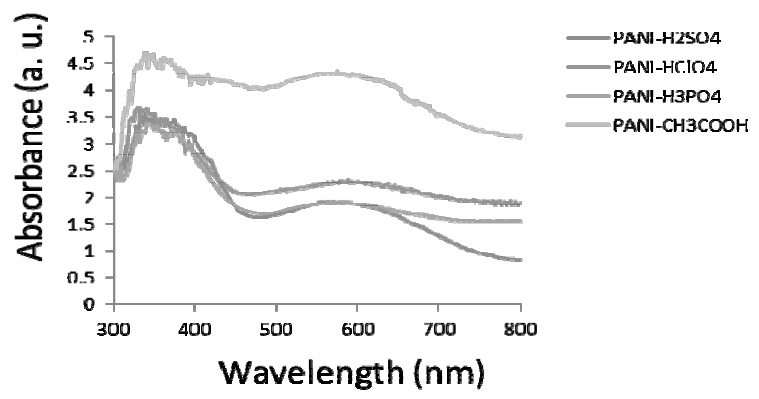

Figure 3: UV-Visible spectrum of PANi samples

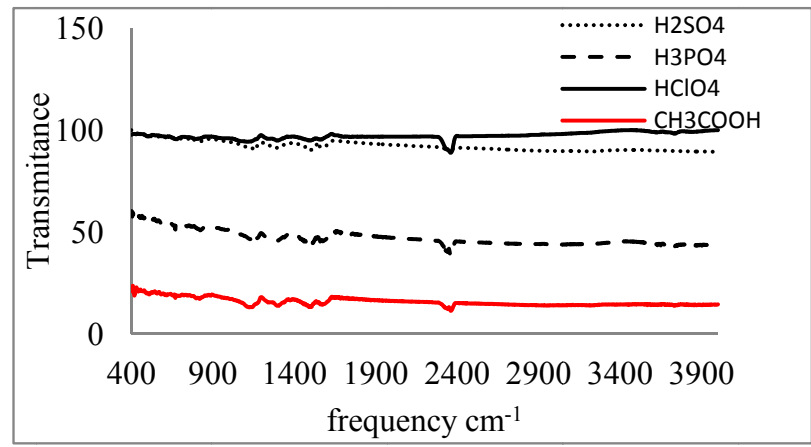

Figure 4: FTIR spectrum of PANi samples 

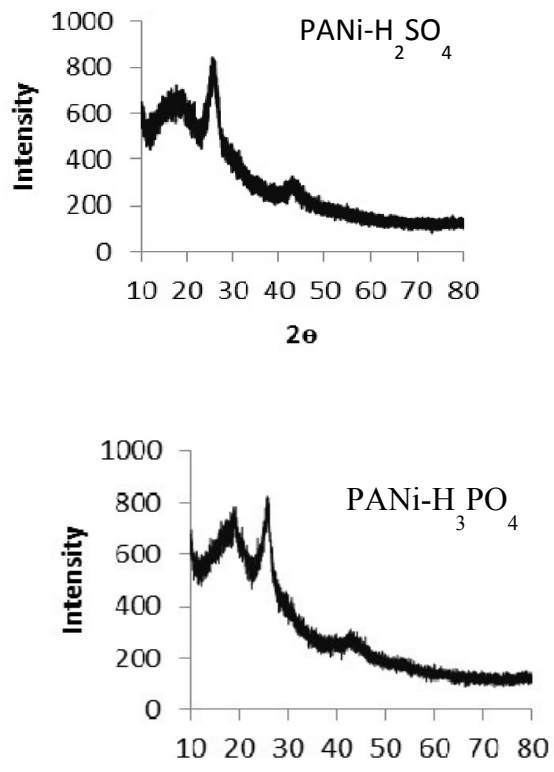

$2 \theta$
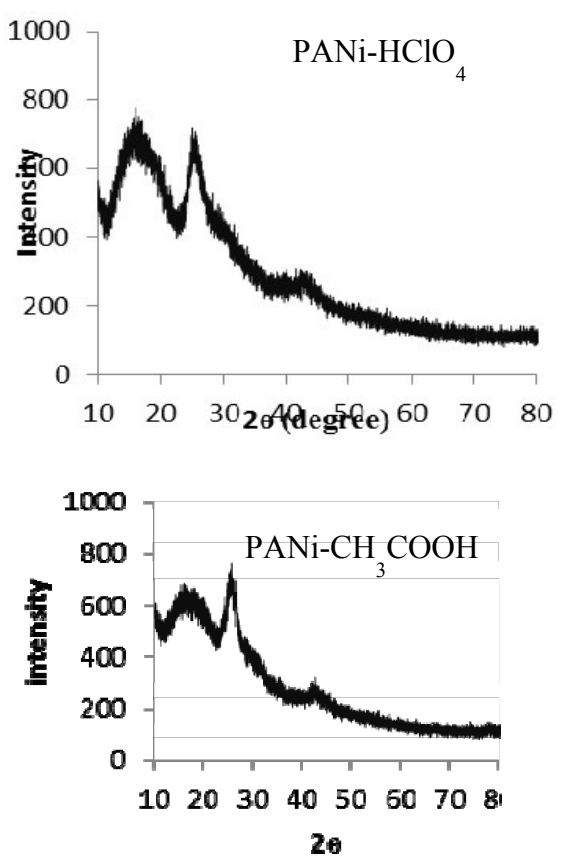

Figure 5: XRD pattern of PANi Samples

\section{Conclusions}

In this work, aniline was polymerized by chemical oxidation method, using ammonium persulphate as an oxidant in $\mathrm{H}_{2} \mathrm{SO}_{4}$ solution. . Composites of various sample of PANi were made with multiwall carbon nanotube and paraffin for the preparation of electrode to study electrochemical properties such as $\mathrm{CV}$ and charge discharge cycle. Electrochemical measurements like cyclic voltammetry and charge-discharge curve of the composite materials were studied which shows that the specific capacitance for the sample (doped with $\mathrm{H}_{2} \mathrm{SO}_{4}$ ) has highest values of $362.12 \mathrm{Fg}^{-1} \mathrm{obtained} \mathrm{from}^{-1}$ $\mathrm{CV}$. The highest specific capacitance value from charge discharge curve was $382.29 \mathrm{Fg}^{-1}$ for $_{2} \mathrm{H}_{2} \mathrm{SO}_{4}$. Composite sample of PANi- $\mathrm{H}_{2} \mathrm{SO}_{4}$ would be best super capacitance than other acid doped PANi.

\section{Acknowledgements}

The authors would like to acknowledge Department of Chemistry, Trichandra Mutiple Campus and Central Department of Chemistry, Tribhuban Univesity, Kathmandu, Nepal for providing the research facilities for conducting the research works.

\section{References}

1. E. Hur and A. Arslan An International Journal of Electrochemmical Science, 2012, 7, 12558- 12572

2. S. Radhakrisna, R. Muthukannan, U. Kamatchi, C.R.K. Rao and M. Vijayan, Indian Journal of Chemistry, 2011, 50, 970-978

3. D. Ghosh, S. Giri, S. Kalra and K.C. Das, Open Journal of Applied Sciences, 2012, 2, 70-77

4. S. Giri, K. C. Das and S. S. Kalra Journal of Materials Science Research 2012, 1, 3

5. Leila Nikza, M.R.Vaezi and B.Yazdani International Journal of Modern Physics 2012, 5, 527-535 